\title{
Computer vision for wildfire research: an evolving image dataset for processing and analysis
}

\author{
Tom Toulouse ${ }^{a} \quad$ Lucile Rossi $^{a} \quad$ Antoine Campana $^{a}$ \\ Turgay Celik ${ }^{b} \quad$ Moulay A. Akhloufi ${ }^{c}$
}

\author{
${ }^{a}$ UMR CNRS 6134 SPE, University of Corsica, 20250 Corte, France \\ ${ }^{b}$ School of Computer Science, University of the Witwatersrand, \\ Johannesburg, South Africa \\ ${ }^{c}$ Computer Science Department, University of Moncton, Moncton, NB, \\ Canada \\ \{toulouse,lrossi,campana\}@univ-corse.fr \\ celikturgay@gmail.com \\ moulay.akhloufi@umoncton.ca
}

\begin{abstract}
The last decade has witnessed the use of computer vision for wildfire detection and measurement. The first and most important step for computer vision analysis is the fire pixel detection because it determines the accuracy of the following processing. The evaluation and the comparison of the wildfire detection algorithms of the literature and the development of new ones needs open datasets with a large number of annotated images and their ground truth. We address this issue by presenting a publicly evolving wildfire annotated image database with ground truth data with examples of use. Currently, it contains 500 visible images and, in a more limited number, multimodal images and videos with frame by frame annotations. This is currently the largest dataset released in this research field.
\end{abstract}

Keywords: Database; image; wildfire; pixel detection;

\section{Introduction}

Wildfires are among the major risks to humans and wildlife around the world $[26,11,10,13]$. Thus, efficient fire detection and behavior anticipation 
systems play an important role in the reduction of destruction caused by fires. The last decade has witnessed the use of computer vision for efficient fire detection $[22,35,40]$, early fire suppression $[19,14,25]$, fire measurement, and fire behavior analysis and prediction [21, 30, 32]. The first and most important step for computer vision analysis is the fire pixel detection because it determines the accuracy of the following processing.

Fire emits radiations in a large spectral band $([0.4 ; 14] \mu \mathrm{m})$. The visible domain $([0.4 ; 0.7] \mu \mathrm{m})$ is the reference domain used in wildland fire research because of the operational simplicity of visible cameras, their very affordable price, and the large quantity of published work using this spectrum. Fire pixel detection on color images is a challenging task because the images are highly affected by the environmental and physical conditions. The main difficulties encountered by the detection methods are due to fire color and the presence of smoke. Indeed, the color can be inhomogeneous (varying from yellow to red color), can have different luminance (depending on the background and the luminosity), and the smoke can mask the fire areas. Several fire detection algorithms working with color images are proposed in the literature [6]. A first category of methods uses color rules. The most commonly used color system is RGB $[2,5,9,17,27,36]$. Other systems are also exploited. Among these systems are those permitting the extraction of luminance-chrominance components, such as YCbCr [4, 41, 34],YUV [20] and $L^{*} a^{*} b^{*}$ [3]. There are also work using so-called cylindrical systems such as TSI [15] and TSV [18]. Finally, some algorithms use combinations of different color spaces $[7,8,31]$. A second category of pixel fire detection algorithms uses machine learning [4, 8, 9, 27, 39]. These detections need to learn from a dataset containing fire pixels and non fire pixels obtained from a sampling of the test image database. In this case, it is important to have a database including a large number of heterogeneous fire color images. To complete this part, several fire detection algorithms use motion analysis [41] to consider or delete fire pixel candidates selected using color criteria. Three works present the comparison of the performances of fire pixel detection algorithms on datasets of wildfire images $[9,34,38]$. In [9] and [34], the images used to benchmark the methods are mainly from two web databases (ForestryImages.org [12] and WildlandFire.com [42]). As there is no a public ground truth (fire contour area obtained manually) associated with each image in these databases, it is impossible to evaluate independently the metrics and the algorithms used in these works. Moreover, the number of different wildfire images of these databases is insufficient to obtain a wildfire pixel representative learning dataset.

Infrared images are easier to process than visible images because the 
intensity of the fire pixels is much higher than the one of the other pixels [21]. The approach to detect the fire zones in an infrared image is to find the threshold that differentiates the pixels belonging to the fire to those of the background. Several threshold search algorithms that can be applied to the detection of fire pixels are proposed in the literature $[23,28,16$, $22]$. The difficulty to consider infrared images is that areas similar to those of fire corresponding to hot gases can also be present in the images and consequently can produce a difference between the fire areas appearing in the visible domain and those of the infrared domain. Works developed by Rossi et al. [33] show that the near infrared domain $([0.75 ; 0.9] \mu \mathrm{m})$ produces wildfire areas that are very similar to the ones obtained in the visible domain. Tacking into account the fact that it is easier to detect fire pixel in infrared images but that the visible images remains the reference, new fire pixel detection algorithms could be develop by using image fusion $[37]$.

The development of the research on wildland fire pixel detection algorithms needs a publicly available database containing a large number of images of wildfire, showing various dominant fire color, conditions of smoke, environments, background, luminosity characteristics, other similar to fire color elements (cars or firefighters for example), and ground truth data. Thus, it is important to be able to evaluate the robustness of a criterion of pixel detection or of an overall method according to isolated parameters like the dominant color or the texture of the fire, the presence and the type of smoke, the background luminosity or the presence of objects that can produce error detections. Similarly, the comparison of the performances of different algorithms must be done considering common criteria and publicly ground truth. Finally, in order to develop new algorithms based on image fusion and pixels movements, multi-modal images and sequences of wildfire have to be also present in this database. In this sense, the present work aims to bring a public test database called Corsican Fire Database (CFDB) and presents its use. The dataset consists of 500 visible images of wildfire collected worldwide, 100 multi-modal (visible and near infrared) images, and 5 sequences of about 30 multi-modal images of outdoor experimental fires captured by the authors. Each image is associated with a black and white (binary) ground-truth image, annotations and descriptors. This database is an evolving one, as its content increases with the images that are deposited online.

The paper is organized as follows. Section 2 gives information about publicly fire image datasets. Section 2.1 informs about the origin of the visible images of the Corsican Fire Database, their selection and the acqui- 
sition protocol of the multi-modal fire images and sequences. Section 2.2 presents the scheme of the manual annotation of the full collection of the database and the descriptors obtained by using image processing. Section 2.3 describes the handling of the new database. The way by which the data associated to the images are made available is described. Information is also given concerning how users of the database can create their own test subset selections for their specific research purposes. Finally, the conclusion appears in section 4 with a summary of the main characteristics of this database and the prospects for its future extensions.

\section{Wildland fire image dataset}

Our research has shown that there was no large public database for wildland fire images. The vast majority of the research uses Internet collected images $[12,42]$ or in house developed datasets of fire images non-available publically. This makes it very difficult to benchmark the different algorithms developed for the study of forest fires. A recent work by Bedo et al. [1] permitted the development of a Flickr-based Fire database. It contains about two thousand pictures; half of them have fire flames in different environments (vegetation, urban, car, etc.). These images were taken from Flickr (a web site for picture and video sharing). The database is under the Creative Commons license that guarantees free public use. Each image has been annotated manually as "fire" or "non fire" by seven human experts but no specific information about the fire pixels areas is given.

Another database is Dyntex [24]. This database is very well organized and its contents properly characterized. It offers a collection of six hundred and fifty high quality dynamic video texture sequences. The sequences contain images of size $720 \times 576$, with a frame rate of 25 images per second, and a duration of at least ten seconds. Each sequence comes with general information (name, date, place, etc.), information about acquisition conditions (camera settings, indoor or outdoor) as well as information about the image texture properties in the sequence (dynamic and spatial properties). However, this database does not contain wildland fires.

Considering what was interesting in the cited datasets and what was to improve, the Corsican Fire Database was developed. It contains images captured in the visible and near infrared spectrum, video sequences, annotation and details about the image characteristics, the environment, etc. Additionally it was built to be an evolving database ready for outside contributions. 


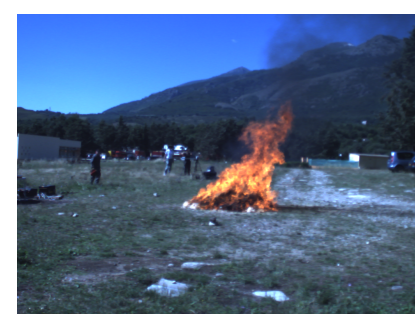

$(a)$

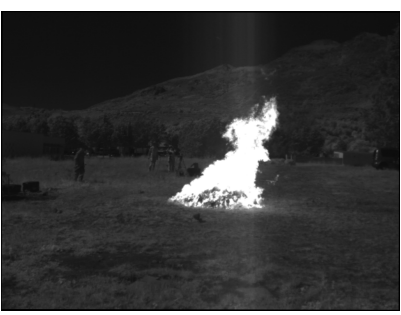

(b)

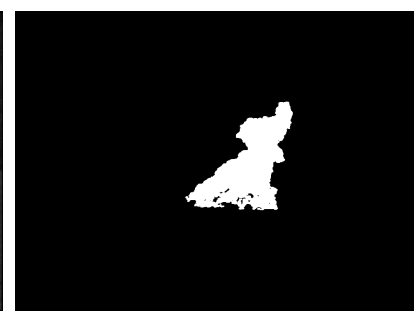

$(c)$

Figure 1: Example of images of the database taken with the multi-spectral camera. ( $a$ ) image of the visible spectra, $(b)$ image of the near infrared spectra and $(c)$ ground truth based on the image $(a)$.

\subsection{Origin of the images}

In order to build the database, a call for wildland fire images was made and more than 2000 images captured on the visible spectra were collected from partners and researchers. These images came from different parts of the world, have different formats and were acquired from cameras with different parameters. 500 images were selected in this set in order to have heterogeneous fire colors and textures, environments, light conditions and vegetation. For each image, a ground truth was built with a manual segmentation of the fire in the image by an expert. This part of the database was used in [39].

The database also contains 100 multi-modal fire images and 5 multimodal sequences of fire in propagation. The multi-modal images were obtained using the JAI AD-080GE camera. This prism based 2-CCD multispectral camera acquires simultaneously an image in the visible spectra and an image in the near infrared (NIR) spectra (700 nm - $900 \mathrm{~nm}$ ) through the same optic. An example of visible and near infrared images taken simultaneously is shown in Fig. 1 (a) and (b). The multi-modal images obtained directly from the JAI AD-080GE camera are not aligned due to the fact that the visible and NIR sensors are not exactly co-aligned. An image registration based on homography matrix transform computation was done in order to align multi-modal images that are available in the database. The camera shutter time was chosen according to the environment luminosity and the focal length was set to $6 \mathrm{~mm}$. The sequences were kept with a frame rate of 1 fps. Images of both spectrum have a size of $1024 \times 768$ pixels.

All the images of the database are in a lossless png format. 


\subsection{Image descriptors}

Each image of the database is annotated using several descriptors. Some of them were annotated manually and others automatically using an image processing procedure. The descriptors are divided into two main categories, global descriptors and fire and environment descriptors. The annotations have two purposes: (i) they can assist users in retrieving particular properties; for example, fires with a particular Color or Texture and (ii) it allows the user to quickly tailor test sets for specific research purposes; for example, to test or develop detection algorithms for specific fire conditions. A number of tools to assist in this selection process are described in Section 5. Some descriptors are missing if this information was not given by the image owner.

\subsubsection{Global descriptors}

The global descriptors give general information on the image. They are listed and described in Table 1. They are separated in two sub-groups, General information and acquisition settings.

The General information are administrative descriptors such as a unique identifier, the sequence number and the image number in the sequence (which are non-zero if the image belongs to a sequence). Another annotations list information on location and date. The last descriptors of this sub-group give the names of the visible image, the associated infrared image (if it exists) and the ground truth image.

The Acquisition settings fields give information about the equipment and settings used to obtain the images: the descriptor $<$ Material $>$ gives the camera model used, <Focal $>$ indicates its focal length , $<$ Sensibility $>$ presents its ISO sensitivity and $<$ Exposure $>$ contains the exposure time used.

If an infrared image is associated to a visible image, the field $<$ Spectra $>$ indicates its spectral domain: near-infrared $(N I R)$, short wavelength infrared $(S W I R)$, medium wavelength infrared $(M W I R)$ or long wavelength infrared $(L W I R)$.

\subsubsection{Fire and environment descriptors}

The purpose of the proposed database is to specify the different parameters of the fire and the environment that can be important for detection purposes. These annotations are listed in Table 2 and are separated in two groups. The Fire descriptors group concerns the annotations specific to fire pixels and 
Table 1: Global properties descriptors. Listed are the field variables, their type and a description of their meaning.

\begin{tabular}{|c|c|c|}
\hline \multicolumn{3}{|c|}{ General information } \\
\hline$<\operatorname{Id}>$ & Number & Unique image identifier \\
\hline$<$ Sequence $>$ & Number & Sequence number \\
\hline$<$ NumIm $>$ & Number & Image number in the sequence \\
\hline$<$ Owner $>$ & Text & Author of the image \\
\hline$<$ Date $>$ & Text & Date of the acquisition \\
\hline$<$ GPS $>$ & Text & GPS position \\
\hline$<\mathrm{Place}>$ & Text & Name of the place of shot \\
\hline$<$ Region $>$ & Text & (Region, Country) of shoot \\
\hline$<$ Name Vis $>$ & Text & Name of the visible spectra image \\
\hline$<$ Name $\operatorname{IR}>$ & Text & Name of the associated IR spectra image (if exists) \\
\hline$<$ Name GT $>$ & Text & Name of the corresponding ground truth image \\
\hline \multicolumn{3}{|c|}{ Acquisition settings } \\
\hline$<$ Material $>$ & Text & Camera model \\
\hline$<$ Focal $>$ & Number & Focal length of the visible camera \\
\hline$<$ Sensibility $>$ & Number & ISO Sensitivity \\
\hline$<$ Exposure $>$ & Number & Exposure time \\
\hline$<$ Spectra $>$ & NIR/ & IR spectral domain (if IR image exists) \\
\hline & SWIR/ & \\
\hline & MWIR/ & \\
\hline & LWIR & \\
\hline
\end{tabular}

the Background descriptors group is about environment pixels. Most of these data were computed automatically with image processing algorithms and the ground truth images. The description and the computation of these descriptors are explained in the following.

Concerning the Fire descriptors:

The $<$ Occupation $>$ field is the percentage of fire pixels in the image. It is computed by dividing the number of fire pixels (pixels labeled "fire" in the ground truth image) by the total number of pixels of the image. In the Table 2, $[0-100]$ indicates that this indicator is between 0 and 100 .

The $<$ Color $>$ field indicates the dominant color of fire pixels in the image. The possible value of this descriptor is Red, Orange, Yellow-White and Other. Each fire pixel is labelled to one of these colors using the HSI color space (see [38] for computation details). The dominant color is the color of the majority of fire pixels. An example of images of the database CFDB with different principal color is shown in Fig. 2.

The $<$ Smoke Superposition $>$ field indicates the percentage of fire pixels which are superimposed with smokes. A learning has been done with support vector machine in order to automatically classify fire pixels with 
Table 2: Fire and background descriptors. Listed are the field variables and their possible values.

\begin{tabular}{ll}
$\begin{array}{l}\text { Fire descriptors } \\
<\text { Occupation }>\end{array}$ & {$[0-100]$} \\
$<$ Color $>$ & Red/Orange/Yellow-White/Other \\
$<$ Smoke Superposition $>$ & {$[0-100]$} \\
$<$ ColorSmoke $>$ & Black/Grey/White \\
$<$ Texture $>$ & $0 / 1$ \\
$<$ Dist F/C $>$ & Near/Far \\
$<$ Direction $>$ & Right/Left/Moves close/Moves away \\
Background descriptors & \\
$<$ Luminosity $>$ & {$[0-100]$} \\
$<$ Time $>$ & Day/Night \\
$<$ Vegetation $>$ & Crimp wood/Low maquis shrubland/High maquis \\
& shrubland/Trees \\
$<$ Presence $>$ & Men/Trunk/None/Other \\
$<$ Clouds $>$ & $0 / 1$ \\
\hline
\end{tabular}

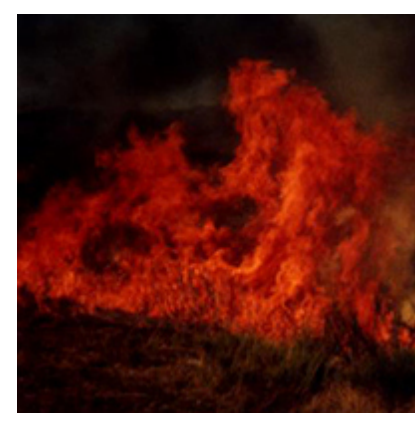

$(a)$

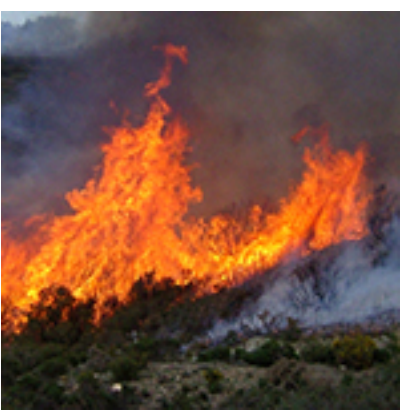

(b)

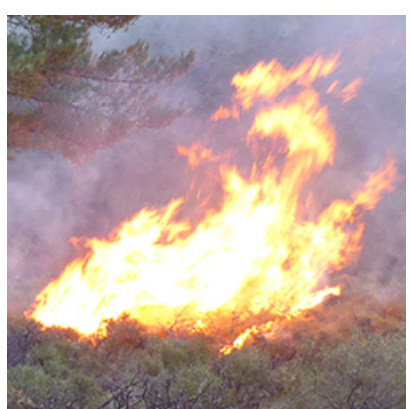

(c)

Figure 2: Example of images of the database that have different value for the $<$ Color $>$ descriptor. The value computed for these images are Red for (a), Orange for (b) and Yellow-White for $(c)$. 


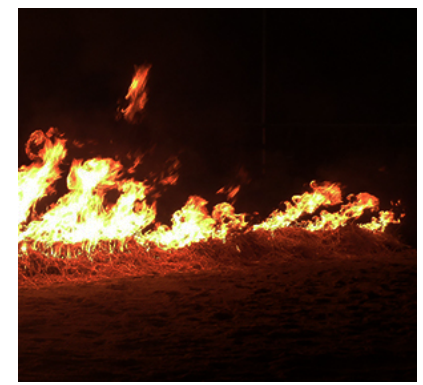

(a)

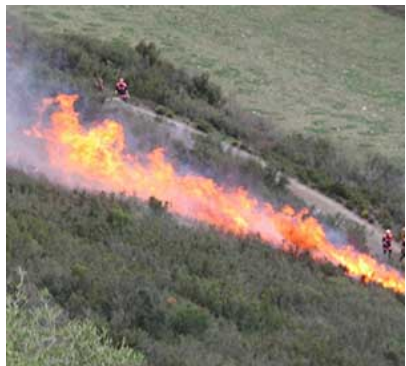

(b)

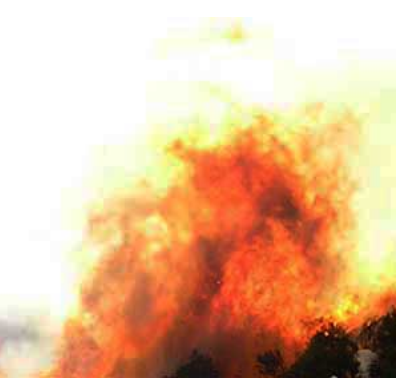

(c)

Figure 3: Example of images of the database that have different value for the $<$ Luminosity $>$ descriptor. The value computed for these images are 10 for $(a), 44$ for $(b)$ and 64 for $(c)$.

and without smoke. The field $<$ ColorSmoke $>$ indicates the color of the smoke. Its value can be Black, Gray or White and is annotated manually.

The $<$ Texture $>$ field indicates if the fire is textured or not accordingly to the entropy of fire pixels (see [38] for computation details). The value "0" informs that the fire is not textured and the value " 1 " indicates that it is textured. For example, the fire area that appears in Fig. $2(b)$ is considered textured unlike the fire region that appears in the Fig.2 $(c)$.

The $<$ Dist $\mathrm{F} / \mathrm{C}>$ field gives an indication on the distance from the fire with respect to the camera. Its value can be near or far. A fire is considered near to the camera when the distance is lower than 200 meters. This field is completed manually, given by the owner of the image from observation or field measurement.

The $<$ Direction $>$ field indicates the direction of the fire compared to the camera. This field is completed manually and given by the owner of the image. The possible values are: right, left, moves close and moves away.

Concerning the background descriptors:

The field <Luminosity $>$ gives an information relating to the brightness of the environment. An automatic procedure computes an average of channel $I$ of $H S I$ color space of the non-fire pixels (see [38] for computation details). The value of this field is a value between 0 and 100. Fig. 3 shows three images that have different luminosity values.

The following descriptors are annotated manually.

The field $<$ Time $>$ indicates if the picture has been captured during the day or the night.

The $<$ Vegetation $>$ field gives information about the vegetable fuel that 
appears in the image. The possible values are: crimp wood, low maquis shrubland, high maquis shrubland and trees.

The $<$ Presence $>$ descriptor indicates the presence of potential false positives area like the ones corresponding to fire men or trucks.

Finally the field $<$ Clouds $>$ indicates if clouds are visible in the sky that can also generate false fire detections especially when processing infrared images. The value "1" indicates the presence of clouds and the value "0" their absence.

\subsubsection{Distribution of the dataset pixels}

The Table 3 gives the image pixel distribution by category. It shows the heterogeneity of the pixels in the dataset.

\begin{tabular}{|c|c|c|c|c|}
\hline & & & Number of pixels & Percentage \\
\hline \multirow{9}{*}{ 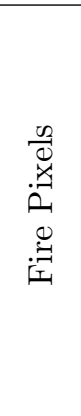 } & \multirow{2}{*}{ Red } & Smoke & 19334066 & $8.1 \%$ \\
\hline & & No Smoke & 43373644 & $18.2 \%$ \\
\hline & \multirow{2}{*}{ Orange } & Smoke & 32812298 & $13.8 \%$ \\
\hline & & No Smoke & 115227509 & $48.4 \%$ \\
\hline & \multirow{2}{*}{$\begin{array}{l}\text { White- } \\
\text { Yellow }\end{array}$} & Smoke & 18519389 & $7.8 \%$ \\
\hline & & No Smoke & 2426830 & $1.0 \%$ \\
\hline & \multirow{2}{*}{ Other } & Smoke & 578832 & $0.2 \%$ \\
\hline & & No Smoke & 5332683 & $2.2 \%$ \\
\hline & & All & 237950619 & $100 \%$ \\
\hline \multirow{4}{*}{ 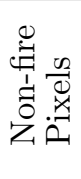 } & \multicolumn{2}{|c|}{ Low Intensity } & 170088686 & $21.9 \%$ \\
\hline & \multicolumn{2}{|c|}{ Medium Intensity } & 259874877 & $33.5 \%$ \\
\hline & \multicolumn{2}{|c|}{ High Intensity } & 345107529 & $44.5 \%$ \\
\hline & \multicolumn{2}{|c|}{ All } & 775071092 & $100 \%$ \\
\hline
\end{tabular}

Table 3: Distribution of the dataset pixels by category

\subsection{Database on Internet}

The Corsican Fire Database is available online at the following URL: http: $/ / c f d b . u n i v-c o r s e . f r /$. After registration, the images and sequences can be downloaded for research purposes via a customized interface. It gives to the user the possibility to download the entire database or to choose specific elements according to the descriptors detailed in the previous section. A screenshot of the browsing interface is shown in the upper part of the Fig 4.

The values of the Occupation, Luminosity and Smoke Superposition descriptors are in the interval [0-100]. The research procedure is carried out 


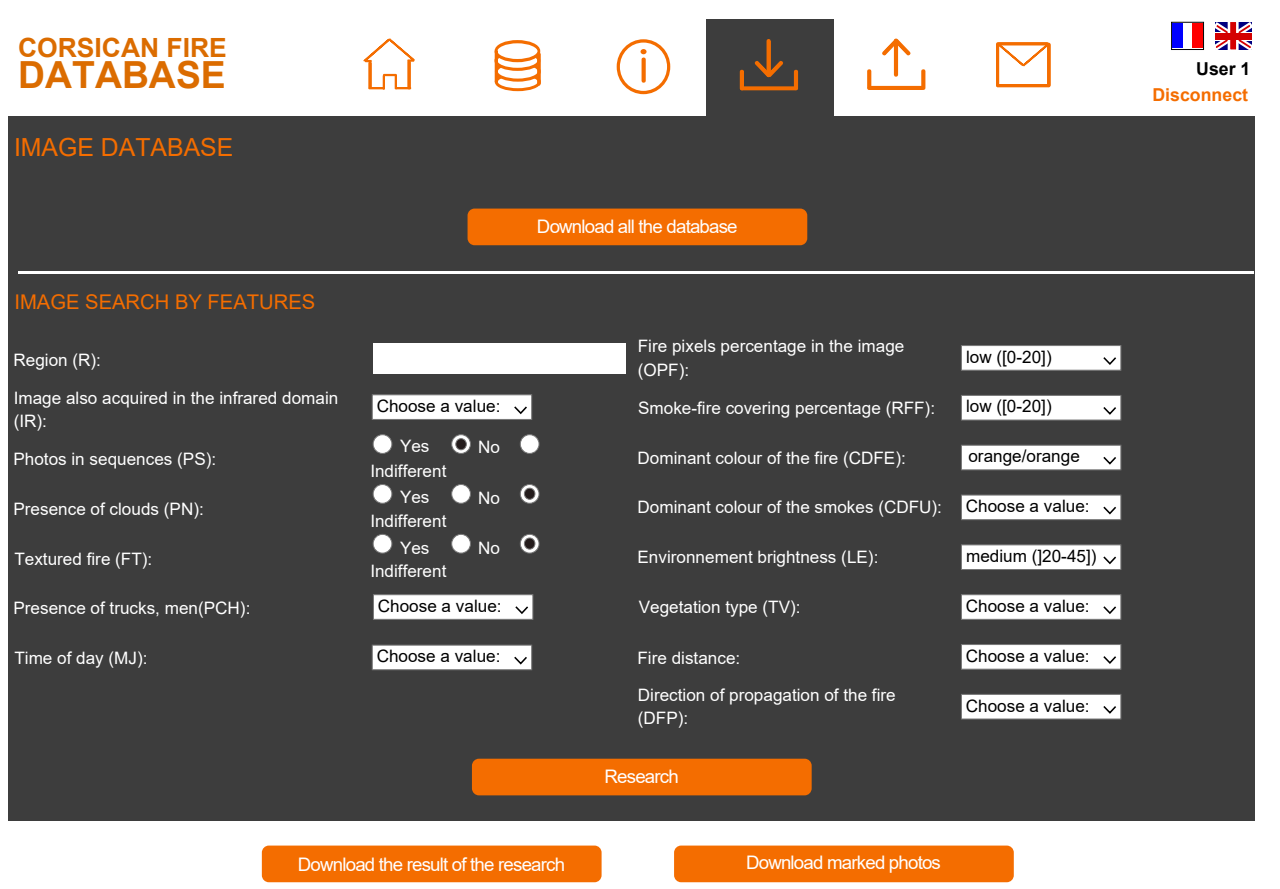

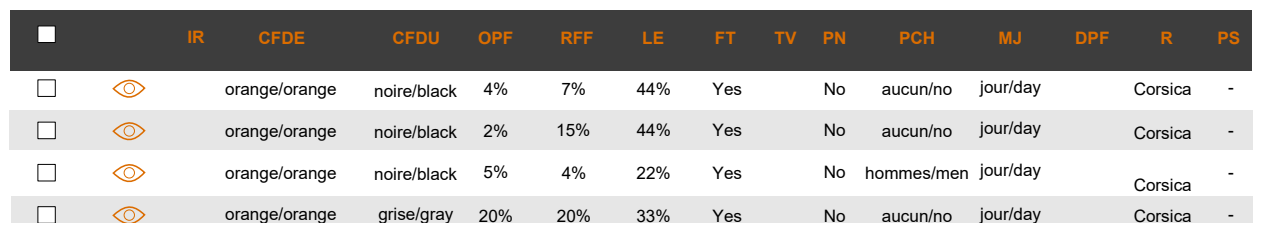

Figure 4: Screenshot of the Corsican Fire Database browser.

by considering three sub-intervals that are: [0-20] (low), ]20-45] (medium) and ]45-100] (high).

The lower part of the Fig 4 shows part of the list of the information associated to the images selected with the following attributes: no specific region, no associated infrared image, not belonging to a sequence, without clouds, not textured, no specific false positive elements, no specific time, low percentage of fire pixels in the image and smoke-fire covering, dominant fire color that is orange, no specific smoke color, with a medium environment brightness, no specific vegetation type, no specific fire distance and no specific direction of fire propagation.

The browsing interface allows to download all the selected images or to select images by considering their information. By clicking on the eye icon it is possible to see the fire image and the associated IR image if it exists. 


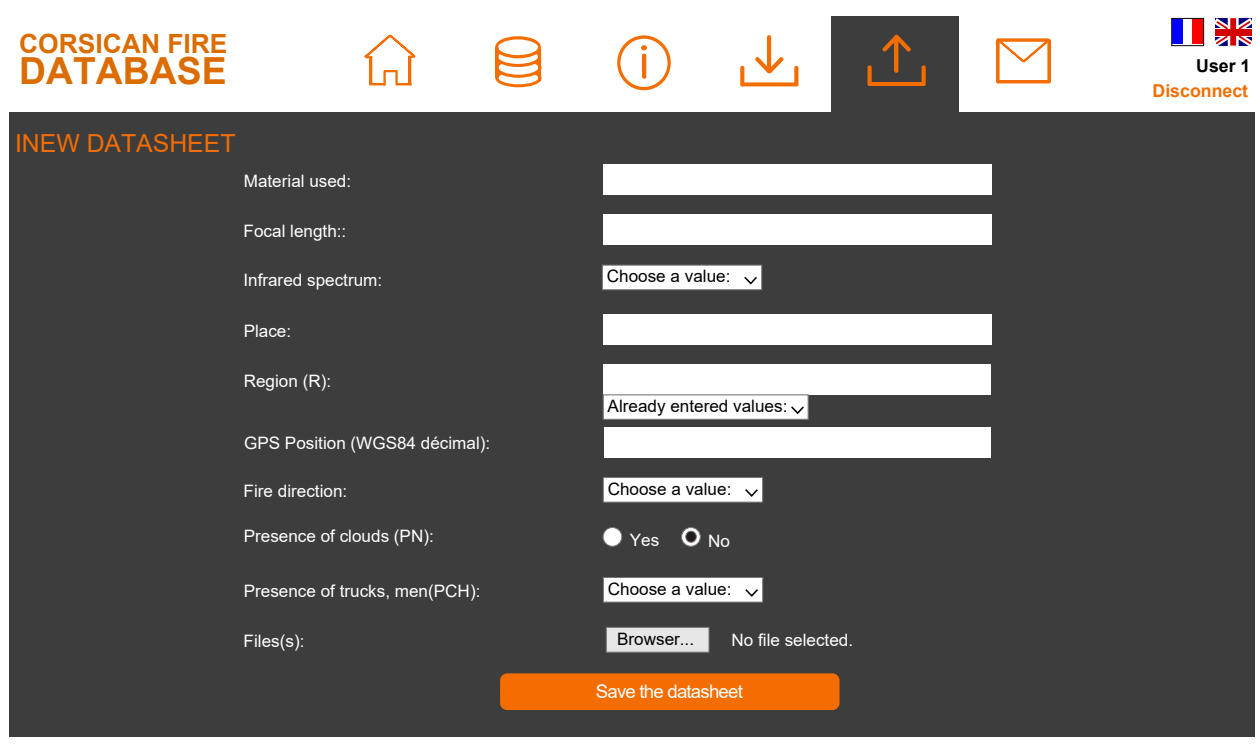

Figure 5: Screenshot of the Corsican Fire Database browser page to upload images.

The user downloads a compressed repository that gathers folders and a csv file. A folder contains at least one visible image and its corresponding ground truth. It can also contain the associated infrared image if it exits. It has all the images (visible, ground truth and infrared) of the same sequence. The $c s v$ file gives all the descriptor values of each image identified by a specific number.

In order that this database evolves over time, the website proposes an interface for the upload of new images or sequences and the recording of the associated data (Fig 6). These resources are not directly integrated with the database and follow a procedure of validation and image processing before their publication on the website. Thus, the database can grow in the future and contain more wildland fire images through users contribution.

\section{Experimental setup}

This section presents examples of processing and analysis that can be carried out using the dataset. 


\subsection{Construction of a pixel learning set}

Some pixel detection methods like $[27,17,4]$ needs labeled fire pixels for training. Following is the description of the method we have used in building the pixels learning set. Fifty images of the dataset were chosen randomly among the five hundred images. The fire pixels of these fifty images were sorted in six categories depending to the color of the pixels (red, orange, white-yellow) and the presence of smoke. The non fire pixels were classified using three levels of intensity (low, medium and high). 500000 non fire pixels and 500000 fire pixels were chosen as follows. For each category, each pixel was represented with a feature vector constructed from color features extracted using different color spaces. An average feature vector was computed for each category and the pixels were sorted based on the distance between their feature vectors and the average feature vector. The pixels were then sampled uniformly to obtain the desired number for each category. According to the observations of Table 3, the pixel distribution was built as follows: $50 \%$ of orange pixels, $33 \%$ of red pixels and $17 \%$ of whiteyellow pixels for fire pixels, and $20 \%$ of low intensity pixels, $40 \%$ of medium intensity pixels and $40 \%$ of high intensity pixels for non-fire pixels. To make it convenient for the processing step, the learning pixels can be organised in order to create an image of size $1000 \times 1000$ whose upper half corresponds to the "fire" pixels and the lower half corresponds to the "non-fire" pixels. This image presented in Fig 6 shows the different categories: region $\mathrm{N}^{\circ} 1$ corresponds to the pixels "red with smoke", region $\mathrm{N}^{\circ} 2$ contains the pixels "red without smoke ", region $\mathrm{N}^{\circ} 3$ includes the "orange with smoke" pixels, region $\mathrm{N}^{\circ} 4$ corresponds to the "smokeless orange" pixels, region $\mathrm{N}^{\circ} 5$ represent "yellow-white pixels" with smoke and region $\mathrm{N}^{\circ} 6$ corresponds to "smoke-free yellow-white" pixels. We can notice that the "non-fire" pixels of "low" and "medium luminosity" are composed of many pixels with green color (which can be assumed to originate from the vegetation in the images). The "non-fire" pixels with "high brightness" represent the color of the sky and the smoke.

\subsection{Performance analysis of pixel detection methods}

The dataset can be used to analyse the performance of fire pixel detection algorithms based on fire pixels and environment characteristics. The performance of the methods are obtained by considering standard metrics that compare the fire areas obtained by pixel detection to the ones obtained manually (the ground truth). In this paper, the F-score [29] is used. As 


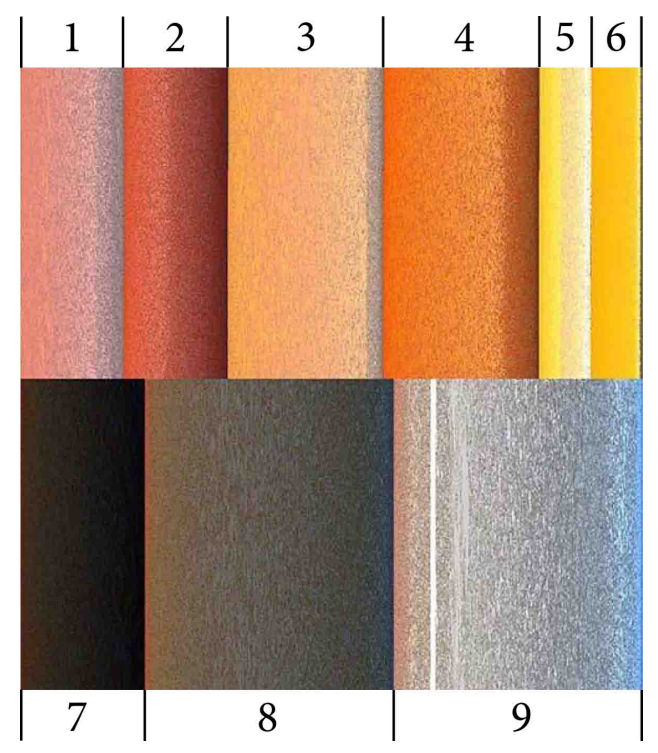

Figure 6: Pixels of the pixel learning set. Nine categories are visible: (1) red with smoke, (2) red without smoke, (3) orange with smoke, (4) orange without smoke, (5) yellow-white with smoke, (6) yellow-white without smoke, (7) low brightness, (8) medium brightness and (9) high brightness.

each tested image has associated descriptors, the scores of pixel detection methods can be obtained according to the images characteristics as it is shown in Table 4 for three different state-of-the-art techniques. From these experimental tests, we can see that for the red colored fires, the technique proposed in [27] is the best performing overall. For the orange colored fires the best performing techniques are [27] and [8]. For yellow fires, [8] is the best performing. The global results on all this colored fires (with and without smoke) show that the best performing technique overall is [27] with an F-score of 0.91 followed by [8] with an F-score of 0.88 .

This data are useful to identify the strengths and weaknesses of the algorithms and to benchmark them. If we need to benchmark fire pixel detection is a specific context images (for example fire pixels with red dominant color and medium smoke), it is possible to get the corresponding set of images using the web interface of the database. 
Table 4: Scores of three state-of-art fire detection methods

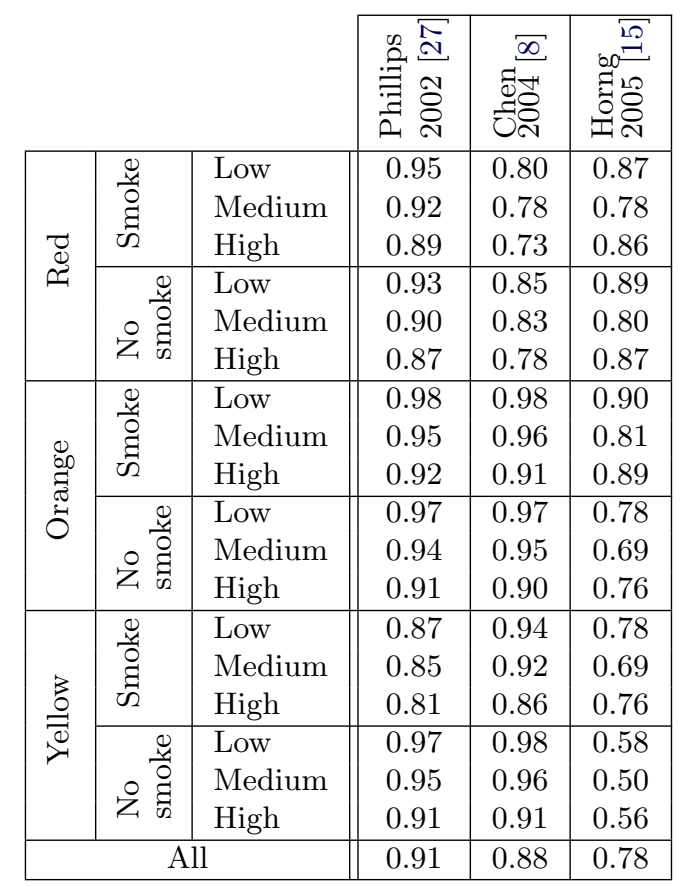

\section{Conclusion}

The Corsican Fire Database aims to provide a common dataset of multimodal wildfire images and videos. This dataset can be used for research and training. It also provides categories of fire and background properties. The proposed wildland fire images database was designed to be an evolving database over time. It contains visible spectrum and near infrared (NIR) images in its current form. Still it was designed to accommodate other spectrums in the future. The visible spectrum color images are by far the most used in current research in the area of forest fires and the proposed database provides a large number of images captured in this spectrum. Additionally, this database contains video sequences captured simultaneously in color and NIR spectrums. These image sequences can serve in the study of multispectral fusion algorithms, the analysis of the performance of fire segmentation in these spectrums, the use of motion for fire segmentation, etc. The userfriendly web interface permits the selection of a subset of images based on different criteria. The users can also contribute to the database by uploading their own images, image sequences (visible, infrared, etc.), corresponding 
ground-truth, and the image parameters. The newly added images are processed using algorithms developed by the authors for further categorization within the database. All these aspects make the proposed database an interesting tool for researchers and professionals in the field of wildland fire study.

\section{Formatting of funding sources}

The present work was supported in part by the French Ministry of Research, the Corsican Region and the CNRS, under Grant CPER 2007-2013.

\section{References}

[1] Bedo, M.V., Blanco, G., Oliveira, W.D., Cazzolato, M.T., Costa, A.F., Rodrigues, J.F., Traina, A.J., Traina, C.: Techniques for effective and efficient fire detection from social media images. ICEIS, p. 3445. (2015)

[2] Borges P.V.K., Mayer J., Izquierdo E.: Efficient visual fire detection applied for video retrieval. In: 16th European Signal Processing Conference, p. 15. IEEE, (2008)

[3] Celik, T.: Fast and efficient method for fire detection using image processing. ETRI journal 32(6), 881-890 (2010)

[4] Celik, T., Demirel, H.: Fire detection in video sequences using a generic color model. Fire Safety Journal 44(2), 147158 (2009)

[5] Celik, T., Demirel, H., Ozkaramanli, H., Uyguroglu, M.: Fire detection using statistical color model in video sequences. Journal of Visual Communication and Image Representation 18(2), 176185 (2007). DOI 10.1016/j.jvcir.2006.12.003

[6] Çetin, A.E., Dimitropoulos, K., Gouverneur, B., Grammalidis, N., Günay, O., Habiboğlu, Y.H., Töreyin, B.U., Verstockt, S.: Video fire detectionreview. Digit. Signal Process. 23(6), 18271843 (2013)

[7] Chen, J., He, Y., Wang, J.: Multi-feature fusion based fast video flame detection. Building and Environment 45(5), 11131122 (2010). DOI 10.1016/j.buildenv.2009.10.017 
[8] Chen, T.H.,Wu, P.H., Chiou, Y.C.: An early fire-detection method based on image processing. In: Image Processing, ICIP04. International Conference on, vol. 3, pp. 17071710. IEEE (2004)

[9] Collumeau, J.F., Laurent, H., Hafiane, A., Chetehouna, K.: Fire scene segmentations for forest fire characterization: A comparative study. ICIP , pp. 29732976 (2011)

[10] EFFIS: European forest fire information system. http://forest.jrc. ec.europa.eu/effis/. Accessed 13 August 2015

[11] FAO: Enlisting communities in wildfire prevention (2010). http:// www.fao.org/news/story/en/item/41230/icode/ Accessed $13 \mathrm{Au}-$ gust 2015

[12] Forestry Images, Forest health, natural resources, fire, trees, wildfire, silviculture photos. http://www.forestryimages.org/. Accessed: 16 May 2016

[13] Fuglem, P.L., Hirsch, K.G., Canadian Council of Forest Ministers: Stratégie canadienne en matière de feux de forêt: synthèses de fond, analyses et perspectives. (2006)

[14] George C.W.and Ewart, G., W.C., F.: Flir: A promising tool for airattack supervisors. Fire Management Notes 50, 26-29 (1989)

[15] Horng, W.B., Peng, J.W., Chen, C.Y.: A new image-based realtime flame detection method using color analysis. In: Networking, Sensing and Control, Proceedings. IEEE, pp. 100105 (2005)

[16] Kittler J., Illingworth J.: Minimum error thresholding. Pattern recognition, 19(1) p. 4147, (1986)

[17] Ko, B.C., Cheong, K.H., Nam, J.Y.: Fire detection based on vision sensor and support vector machines. Fire Safety Journal 44(3), 322329 (2009)

[18] Liu C.B., Ahuja N.: Vision based fire detection. In: Proceedings of the 17th International Conference on Pattern Recognition,(ICPR), volume 4, p. 134137. IEEE, (2004)

[19] Loane I.T.and Goul, J.: Aerial suppression of bushfires: cost-benefit study for Victoria (1986) 
[20] Marbach G., Loepfe M., Brupbacher T.: An image processing technique for fire detection in video images. Fire safety journal, 41(4) p. 285289, (2006)

[21] Martínez-de Dios, J.R., Merino, L., Caballero, F., Ollero, A.: Automatic forest-fire measuring using ground stations and unmanned aerial systems. Sensors 11(6), 63286353 (2011)

[22] Martínez-de Dios, J.R., Merino, L., Ollero, A.: Fire detection using autonomous aerial vehicles with infrared and visual cameras. IIFAC (2005)

[23] Otsu, N.: A threshold selection method from gray-level histograms. Automatica 11(285-296), 2327 (1975)

[24] Péteri R., Fazekas, S., Huiskes, M.J.: DynTex : a Comprehensive Database of Dynamic Textures. Pattern Recogn. Lett., 31, pp 16271632 (2010) doi: 10.1016/j.patrec.2010.05.009

[25] Pérez, Y., Pastor, E., Planas, E., Plucinski, M., Gould, J.: Computing forest fires aerial suppression effectiveness by IR monitoring. Fire Safety J. 46(1-2), 2-8 (2011). doi: 10.1016/j.firesaf.2010.06.004

[26] Prométhée2 : The database on forest fire in Mediterranean region in France. http://www. promethee.com/default/bilan-tableaux. Accessed 13 August 2015

[27] Phillips III, W., Shah, M., da Vitoria Lobo, N.: Flame recognition in video. Pattern recogn. Lett. 23(1), 319327 (2002)

[28] Ridler T., Calvard S.: Picture thresholding using an iterative selection method. IEEE transactions on Systems, Man and Cybernetics, 8(8), $630632,(1978)$

[29] Van Rijsbergen C.J. Information Retrieval. Butterworth-Heinemann, London, 2nd edition, 1979.

[30] Rodriguez, F., Moreno Robles, A.: D-09-04.The infrared imager: its use for wildland fire monitoring. Tech. rep., EUROFIRELAB (2004)

[31] Rossi, L., Akhloufi, M.: Dynamic fire 3D modeling using a realtime stereovision system. In: M. Iskander, V. Kapila, M.A. Karim (eds.) Technological Developments in Education and Automation, pp. 3338. Springer Netherlands, Dordrecht (2010) 
[32] Rossi, L., Balbi, J.H., Rossi, J.L., Marcelli, T., Pieri, A.: Front fire propagation model: use of mathematical model and vision technology. Advanced Technologies, Research - Development - Applications pp. 745760 (2006).

[33] Rossi, L., Toulouse, T., Akhloufi, M., Pieri, A., Tison, Y.: Estimation of spreading fire geometrical characteristics using near infrared stereovision. In: IS\&T/SPIE Electronic Imaging, pp. 86,500A-86,500A (2013). doi: $10.1117 / 12.2001624$

[34] Rudz, S., Chetehouna, K., Hafiane, A., Laurent, H., Sero-Guillaume, O., : Investigation of a novel image segmentation method dedicated to forest fire applications. Meas. Sci. Technol. 24(7), pp.075403 (2013)

[35] Stipaničev, D. and Vuko, T. and Krstinić, D. and Štula, M. and Bodrozic, L.: Forest fire protection by advanced video detection systemcroatian experiences. TIEMS Workshop-Improvement of Disaster Management System (2006)

[36] Töreyin, B. U., Dedeoğlu, Y., Güdükbay, U., Çetin, A. E., : Computer vision based method for real-time fire and flame detection. Pattern Recognition Letters. 27(1), pp. 49-58 (2006)

[37] Toulouse, T.: Estimation par stéréovision multimodale de caractéristiques géométriques d'un feu de végétation en propagation. $\mathrm{Ph}$.D. thesis, University of Corsica (2015)

[38] Toulouse, T., Rossi, L., Akhloufi, M., Celik, T., Maldague, X.: Benchmarking of wildland fire colour segmentation algorithms. IET Image Process. (2015)

[39] Toulouse, T., Rossi, L., Celik, T., Akhloufi, M.: Automatic fire pixel detection using image processing: a comparative analysis of rule-based and machine learning based methods. Signal, Image and Video Processing pp. 1-8 (2015)

[40] Verstockt, S., Lambert, P., Van de Walle, R., Merci, B., Sette, B.: State of the art in vision-based fire and smoke dectection. In: 14th International Conference on Automatic Fire Detection (2), pp. 285292(2009)

[41] Wang, D., Cui, X., Park, e., Jin, C., Kim, H.: Adaptive flame detection using randomness testing and robust features, Fire Safety Journal. 55(0) pp. 116125 (2013) 
[42] WildlandFire.com, Home of the wildland firefighter. http://www. wildlandfire.com/. Accessed: 16 May 2016 